\title{
Down syndrome associated with father's age in Norway
}

\section{J DAVID ERICKSON AND TOR BJERKEDAL}

From the Bureau of Epidemiology, Public Health Service, Department of Health, Education, and Welfare, Center for Disease Control, Atlanta, Georgia, USA; and the Institute for Hygiene, University of Oslo, Oslo, Norway.

SUMMARY Records of births in Norway in 1967 to 1978 were examined for evidence of an increased $\vec{\sim}$ risk of Down syndrome associated with older paternal age. From among some 685000 total births ${ }_{0}^{N}$

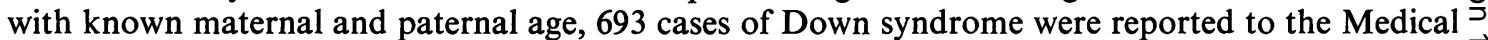
Birth Registry of Norway. The effect of paternal age was assessed by classifying fathers as young and 7 old on the basis of several definitions. The effect of maternal age was removed by stratifying the data $\stackrel{\mathbb{D}}{O}$ on single years of mothers' age. When fathers were considered young if they were $\leq 49$ and old if they were $\geq 50$, the analysis yielded a statistic for the test of a one-sided hypothesis which was significant $之$ at the 0.05 level. There appears to be an increased risk (perhaps 20 to $30 \%$ ) of Down syndrome $\overrightarrow{0}$ associated with older fathers, independent of a maternal age effect. If this increase does in fact exist, $\stackrel{\infty}{-}$ it is much smaller than the increases in risk associated with advancing maternal age, and because 0 older men contribute a relatively small proportion of total births their contribution to the communal burden of Down syndrome is quite small. However, the finding is of aetiological interest and is the first indication of a significant paternal age effect where control for maternal age has been $\stackrel{\unrhd}{\varnothing}$ stringent.

There has been a recent renewal of interest in the possibility that older fathers might be at higher risk of producing babies with Down syndrome. The cytogenetic evidence which has become available during the last few years has been reviewed by Magenis and Chamberlin ${ }^{1}$ and they suggested that a sizable proportion, perhaps as many as 20 to $25 \%$, of Down syndrome cases have their origins in paternal non-disjunction. Is this paternal contribution associated with the father's age, as the maternal contribution is associated with age, or is it not? Stene $e t \mathrm{al}^{2}$ felt that they demonstrated a higher rate of Down syndrome among fathers 55 years of age and older. The study by Matsunaga et $a l^{3}$ did not fully resolve the issue since, while they seem to have found an increased risk among fathers $\geqslant 55$, they also found a significantly reduced risk among fathers of 40 to 44 . One of us ${ }^{4}{ }^{5}$ investigated this question in three separate sets of data and found no evidence in favour of an increased risk among older fathers. However, some of the data used in these latter studies have been criticised, as have some aspects of Received for publication 27 February 1980 the statistical analyses. ${ }^{6}$ On the other hand we have been critical of the less than strict control of maternal age used by others. ${ }^{2} 3$

We report here on another investigation into this question in which we used contemporary Norwegian 3 data. These data do not represent a complete ascertainment of Down syndrome among the total $\frac{3}{3}$ birth population but they nevertheless offer some insights into the problem.

\section{Material and methods}

In Norway each birth (from a pregnancy of $\geq 16^{\circ}$ weeks) is recorded in the Medical Birth Registry. 7 . Our data were from the registry for the period $1967 \mathrm{\omega}$ to 1978 . On each certificate of birth there is provision for recording malformations noted in the baby. A number of other characteristics are also $\stackrel{\Phi}{\Phi}$ noted on each certificate, including maternal age, and, in most instances, paternal age. From various special studies, data on such attributes as maternal $\frac{D}{\mathbb{D}}$ education and employment are available for sub- $\frac{?}{\mathbb{0}}$ sets of the total registry material. 
A number of methods for separating the effects of maternal and paternal age on the frequency of Down syndrome have been used in the past. These methods have been reviewed by Stene and Stene, ${ }^{8}$ who pointed out certain technical deficiencies in them. In addition, they proposed a simple but powerful method for detecting a paternal age effect in the presence of a strong maternal age effect. Their method involves the following proposition: if there is a paternal age effect, it might be similar to the effect of maternal age, that is, relatively constant incidence up to a certain age and then increasing sharply. If the paternal age effect follows this pattern, then the simple expedient of dividing fathers into two groups, young and old, would provide a useful means of detecting the effect, if the proper definitions of young and old are chosen.

In using this technique it is necessary to control for the effect of maternal age, since mothers' and fathers' age are highly correlated and since the incidence of Down syndrome increases dramatically with increases in maternal age. One of us has emphasised the need to provide stringent control of the maternal age effect. ${ }^{45}$ Thus, in the work reported here we have taken account of the effect of the mother's age by single years of age. When set out in this way, the data are in the form of a series of 2 $\times 2$ tables, one for each year of maternal age. On one axis of the tables the dichotomy is father's age, young or old, and on the other axis it is the status of the baby, affected by Down syndrome or not. In each table the association between Down syndrome and father's age can be assessed, and then the results summarised over all tables. Stene and Stene ${ }^{8}$ proposed using Fisher's exact test for assessing each table and then combined the results over tables. Such an approach is not computationally feasible with the Norwegian data, since the numbers of cases and births are large and also because controlling for maternal age by single years creates a large number of tables. Instead, we used the Mantel-Haenszel test $^{9}$ which is a large sample approximation of the test used by Stene et al. ${ }^{2}$ The Mantel-Haenszel test yields a $\chi^{2}$ for each table and a summary $\chi^{2}$ which provides an overall measure of the significance of the paternal age effect. This test compares the observed numbers of Down syndrome cases in each paternal age category with the numbers expected. These expected numbers are based on the numbers of births in each paternal age category and the rate of Down syndrome among young and old fathers combined. Thus, the test assesses the difference between two indirectly adjusted rates ${ }^{10}$ and we present some of the results of our analyses in the form of adjusted rates. Since there is no a priori definition of young and old fathers, we tested the hypothesis of no paternal age effect using several definitions. The data were assessed using each of the following divisions of fathers into young and old groups, $\leq 34-\geq 35, \leq 39-\geq 40$, and so on in 5 -year increments up to $\leq 54-\geq 55$.

In addition to these main analyses, we also tried to determine whether a trend in Down syndrome incidence was associated with increasing paternal age. This was done using Mantel's extension ${ }^{11}$ of the Mantel-Haenszel test, where the effects of maternal age were accounted for by single years of age. Two analyses were done, one where paternal age was treated by single years of age and one where it was grouped into 5-year age intervals.

\section{Results}

ASCERTAINMENT OF DOWN SYNDROME

During the 12-year study period there were 739574 babies born (live and still) in Norway. From among these there were 731 with a diagnosis of Down syndrome, an overall incidence rate of 9.88 per 10000 births. The maternal age specific incidences are shown in the figure. To remove the effects of relatively small numbers of cases available at each maternal age, the rates shown have been smoothed with a 5-year moving average. In order to appreciate the degree of underascertainment of Down syndrome by the Medical Birth Registry of Norway, data from Sweden are also shown in the figure. These Swedish data represent the results of intensive case finding for the years 1968 to 1970 and thus provide a reasonable comparison for the Norwegian data. The data plotted in the figure were taken from table 1 of the paper by Hook and Lindsjö ${ }^{12}$ and were smoothed with a 5-year moving average. Examination of the figure will show that there appears to be underascertainment of Down syndrome by the Norwegian registry at all ages. Moreover, the underascertainment is somewhat more pronounced for babies with younger mothers. Below the maternal age of 25 the Swedish rates are about 1.6 or 1.7 times higher than the Norwegian rates, whereas from maternal age 25 to 42 or 43 the Swedish rates are only 1.3 to 1.4 times higher. For ages above 43 or 44 , the Norwegian rates again appear to diverge more from the Swedish rates, but this may be because of small numbers. This underascertainment introduces the potential for biases which might interfere with assessing the effects of paternal age.

\section{DOWN SYNDROME BY}

MATERNAL EDUCATION AND OCCUPATION

Stene and Stene $^{6}$ have suggested that relatively fewer cases of Down syndrome might be ascertained 


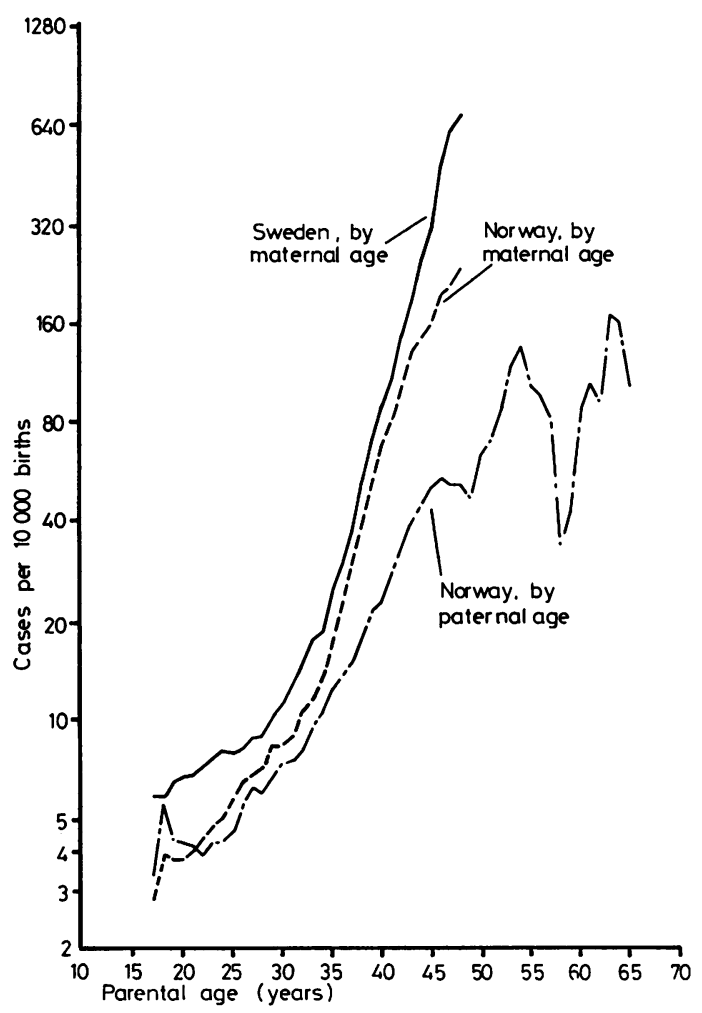

FIGURE Down syndrome rates, smoothed by 5-year moving average, by parental age for Norway 1967-1975 and Sweden 1968-1970.12

in low socioeconomic groups and that this difference in reporting level could interfere with showing an effect of paternal age. We evaluated the occurrence of Down syndrome by level of maternal education in a sub-set of the total material. Table 1 shows that there are no substantial differences for the various education categories. We have also investigated the association between frequency of Down syndrome and various measures of occupational status and found little variation from group to group.

DOWN SYNDROME BY FATHER'S AGE

Paternal information was missing from $5 \cdot 2 \%$ of the certificates of babies with Down syndrome and from $7 \cdot 2 \%$ of certificates of normal babies. This de- ficiency is more pronqunced among the normal babies since paternal information is more frequently
TABLE 1 Adjusted rates*† of Down syndrome by maternal education, Norway, 1970-1973

\begin{tabular}{lc}
\hline Maternal education & Rate \\
\hline Primary school & $11 \cdot 84$ \\
Lower stage & $(66 / 57 \cdot 27)$ \\
Second level & $9 \cdot 12$ \\
First stage & $(97 / 109 \cdot 24)$ \\
Second level & $11 \cdot 99$ \\
Second stage I & $(54 / 46 \cdot 28)$ \\
Second level & $8 \cdot 80$ \\
Second stage II-III & $(21 / 24 \cdot 52)$ \\
Third level & 9.98 \\
& $(23 / 23 \cdot 68)$
\end{tabular}

* Rates adjusted by 5 -year maternal age groupings (per 10000 births). $\uparrow$ Numbers in parentheses are (observed Down syndrome cases/ expected cases).

missing from certificates of births to young mothers $\vec{\oplus}$ than it is from certificates of births to older mothers $\infty$ (for example, 9.0\% among births to women 20 to. 24 years of age vs $2 \cdot 5 \%$ among births to women 40 to 44). We have found that the rate of Down syndrome is about $20 \%$ higher for babies whose father's age is not known than it is for those whose father's age is known. However, this effect is $\stackrel{\circ}{\circ}$ associated equally with young and older mothers $\overrightarrow{\overrightarrow{0}}$ (table 2).

TABLE 2 Adjusted rates*† of Down syndrome by maternal age and known and unknown paternal age, Norway, 1967-1978.

\begin{tabular}{|c|c|c|}
\hline \multirow[t]{2}{*}{ Maternal age } & \multicolumn{2}{|l|}{ Paternal age } \\
\hline & Known & Unknown \\
\hline$\leq 19$ & $\begin{array}{c}3 \cdot 39 \\
(13 / 10 \cdot 97)\end{array}$ & $\begin{array}{c}1 \cdot 68 \\
(3 / 5 \cdot 03)\end{array}$ \\
\hline $20-39$ & $\begin{array}{l}8.07 \\
(517 / 522 \cdot 71)\end{array}$ & $\begin{array}{l}10 \cdot 25 \\
(28 / 22 \cdot 29)\end{array}$ \\
\hline$\geq 40$ & $\begin{array}{l}109 \cdot 68 \\
(163 / 165 \cdot 77)\end{array}$ & $\begin{array}{l}184 \cdot 58 \\
(7 / 4 \cdot 23)\end{array}$ \\
\hline Total & $\begin{array}{c}9.79 \\
(693 / 699.45)\end{array}$ & $\begin{array}{c}11 \cdot 90 \\
(38 / 31 \cdot 55)\end{array}$ \\
\hline
\end{tabular}

* Rates adjusted by single years of maternal age within each maternal group (per 10000 births).

†Numbers in parentheses are (observed Down syndrome cases/胥 expected cases). 
The rates of Down syndrome by single years of father's age are also shown in the figure (smoothed with a 5-year moving average). These data, and all others in our study that deal with paternal age, pertain only to the 693 babies whose father's age was known. The paternal age specific rates closely parallel the maternal age specific rates from the early 20 s to age 35 . At ages over 35 or so the paternal age specific rates rise less rapidly than the maternal age specific rates, and over 45 or 50 the rate of increase is further diminished. In interpreting these

TABLE 3 Mean maternal age by paternal age group, all births, Norway, 1967-1978

\begin{tabular}{lll}
\hline Paternal age & \multicolumn{2}{l}{ Maternal age } \\
\cline { 2 - 3 } & Mean & $S D$ \\
\hline & & \\
\hline 19 & $18 \cdot 77$ & $1 \cdot 61$ \\
$20-24$ & $21 \cdot 48$ & $2 \cdot 28$ \\
$25-29$ & $24 \cdot 86$ & $2 \cdot 83$ \\
$30-34$ & $28 \cdot 40$ & $3 \cdot 40$ \\
$35-39$ & $31 \cdot 88$ & $4 \cdot 12$ \\
$40-44$ & $34 \cdot 91$ & $4 \cdot 70$ \\
$45-49$ & $36 \cdot 90$ & $5 \cdot 19$ \\
$50-54$ & $37 \cdot 82$ & $5 \cdot 54$ \\
$\geq 55$ & $37 \cdot 27$ & $6 \cdot 11$ \\
\hline
\end{tabular}

rates, the correlation between maternal and paternal ages must be taken into account. For each 5-year group of paternal age, table 3 shows the average maternal age and the corresponding standard deviation. As the father's age rises, the corresponding increase in maternal age diminishes. In addition, the standard deviation of maternal age rises with paternal age. This reflects the fact that as men age they have offspring by women of a wider and wider range of ages.

The Mantel-Haenszel tests for a paternal age effect using the dichotomies $\leq 34-\geq 35$ to $\leq 44$ $\geq 45$ yielded insignificant summary $\chi^{2}$ values, as did the test for the dichotomy $\leq 54-\geq 55$. However, the test for the dichotomy $\leq 49-\geq 50$ gave a summary $\chi^{2}$ of 2.81 , which is significant at the 0.05 level if one considers a one-sided test appropriate (that is, a null hypothesis of no higher rate in the older paternal age group). Table 4 presents the adjusted Down syndrome rates for four paternal age categories and 5-year groups of maternal age. The paternal age specific rates were adjusted by single years of maternal age within each 5-year interval of maternal age. All paternal ages $\leq \mathbf{4 4}$ are grouped together since no sub-set of them showed any evidence of a paternal age effect. Overall, fathers of ages 45 to 49 showed a slightly decreased risk while those of 50 years of age and over showed an overall increased risk of 25 or $30 \%$ (table 4 ). The

TABLE 4 Adjusted rates* ${ }^{*}$ of Down syndrome by maternal and paternal age, Norway, 1967-1978

\begin{tabular}{|c|c|c|c|c|}
\hline \multirow[t]{2}{*}{ Maternal age } & \multicolumn{4}{|l|}{ Paternal age } \\
\hline & $\leq 44$ & $45-49$ & $50-54$ & $\geq 55$ \\
\hline$\leq 19$ & $\begin{array}{c}3 \cdot 38 \\
(13 / 12 \cdot 991)\end{array}$ & $\overline{(0 / 0.006)}$ & $\overline{(0 / 0.002)}$ & $\overline{(0 / 0.001)}$ \\
\hline $20-24$ & $\begin{array}{l}4 \cdot 35 \\
(103 / 102 \cdot 834)\end{array}$ & $\overline{(0 / 0 \cdot 125)}$ & $\overline{(0 / 0.027)}$ & $\overline{(0 / 0 \cdot 014)}$ \\
\hline $25-29$ & $\begin{array}{l}6 \cdot 83 \\
(160 / 161 \cdot 009)\end{array}$ & $\begin{array}{c}9 \cdot 28 \\
(1 / 0 \cdot 740)\end{array}$ & $\overline{(0 / 0 \cdot 179)}$ & $\begin{array}{c}95.42 \\
(1 / 0.072)\end{array}$ \\
\hline $30-34$ & $\begin{array}{l}10 \cdot 77 \\
(119 / 116 \cdot 051)\end{array}$ & $\overline{(0 / 2 \cdot 958)}$ & $(1 / 0 \cdot 768)$ & $\overline{(0 / 0 \cdot 223)}$ \\
\hline $35-39$ & $\begin{array}{l}28 \cdot 33 \\
(107 / 109 \cdot 519)\end{array}$ & $\begin{array}{c}33 \cdot 17 \\
(20 / 17 \cdot 486)\end{array}$ & $\begin{array}{c}14.95 \\
(2 / 3.879)\end{array}$ & $\begin{array}{c}77.96 \\
(3 / 1.116)\end{array}$ \\
\hline $40-44$ & $\begin{array}{l}97 \cdot 97 \\
(70 / 72 \cdot 786)\end{array}$ & $\begin{array}{c}92 \cdot 23 \\
(42 / 46 \cdot 388)\end{array}$ & $\begin{array}{l}154 \cdot 44 \\
(24 / 15 \cdot 831)\end{array}$ & $\begin{array}{c}76 \cdot 50 \\
(3 / 3 \cdot 995)\end{array}$ \\
\hline$\geq 45$ & $\begin{array}{l}202 \cdot 68 \\
(5 / 4 \cdot 857)\end{array}$ & $\begin{array}{l}175 \cdot 52 \\
(9 / 10 \cdot 095)\end{array}$ & $\begin{array}{l}202 \cdot 76 \\
(7 / 6 \cdot 797)\end{array}$ & $\begin{array}{l}262 \cdot 39 \\
(3 / 2 \cdot 251)\end{array}$ \\
\hline Total & $\begin{array}{l}10 \cdot 05 \\
(577 / 580 \cdot 047)\end{array}$ & $\begin{array}{c}9 \cdot 35 \\
(72 / 77 \cdot 798)\end{array}$ & $\begin{array}{l}12 \cdot 49 \\
(34 / 27 \cdot 483)\end{array}$ & $\begin{array}{l}13 \cdot 16 \\
(10 / 7 \cdot 672)\end{array}$ \\
\hline
\end{tabular}

* Rates adjusted by single years of maternal age within each maternal age group by the indirect method (per 10000 births).

$\dagger$ Numbers in parentheses are (observed Down syndrome cases/expected cases). 
extended Mantel-Haenszel tests used to assess the data for evidence of a trend in paternal age specific rates did not yield significant statistics.

\section{Discussion}

These data suggest that the risk of Down syndrome may be modestly increased when fathers are over 50 or so years of age. This finding is important since it is the first indication of such an effect found where there has been stringent control of the maternal age effect. A search for a paternal age effect has been made many times in the past, but the hunt was renewed recently when cytogenetic evidence showed that paternal non-disjunction could be a source of trisomy 21. Stene and $\mathrm{Stene}^{8}$ suggested that the statistical procedures used in earlier studies had been weak and proposed a simple but powerful alternative. Using this approach they seemingly showed an excess risk among fathers $\geq 55$ years of age. The results of their study have been questioned, ${ }^{45}$ however, since they controlled for the effect of maternal age by grouping into broad categories. Any such test for a paternal age effect will be clouded to a certain extent by a residual effect of maternal age. In any broad based maternal age grouping, the ages of the mates of younger fathers will tend to cluster around the lower bound of the maternal age category and vice versa. Sved and Sandler ${ }^{13}$ used a computer simulation to show that such a bias will probably not be too great for small samples such as the one used by Stene et al. ${ }^{2}$ Nevertheless, the statistical expectation is one of a contamination of the apparent paternal age effect with a residual maternal contribution. Stene and Stene $^{6}$ suggested that while control of maternal age by single years might be desirable, it has the disadvantage of reducing the statistical power because it involves the estimation of many parameters associated with maternal age. There seems to be no way out of this dilemma, but we are of the opinion that concerns of validity outweigh concerns of power, and that any analytic procedure should yield a result as free of the effects of maternal age as possible. The approach used here, adjusting for maternal age by single years of age, would seem to fulfil that requisite.

Despite the possible reduced power associated with this approach, we have found what appears to be a modest effect of older fathers. This effect is of the order of a $25 \%$ increase in the risk, which is relatively small compared to the maternal age effect. For example, the risk associated with mothers of $\mathbf{4 0}$ and over is about $1300 \%$ higher than that with those under 40. Furthermore, the effect was of marginal statistical significance and there was a small de- ficiency of infants with Down syndrome and fathers aged 45 to 49 . Even so, there is a certain consistency to these Norwegian data since the rates appear to be higher both for fathers of 50 to 54 and for those $\geq 55$ years.

Since the size of the $\chi^{2}$ associated with the paternal age dichotomy $\leq 49$ to $\geq 50$ is not large, one may question how likely it is that chance or bias produced of the observed result. Since interest in this area has $\overrightarrow{0}$ focused on the question of whether older fathers have a higher risk than younger fathers, a one-tailed test seems appropriate. Thus our finding of a higher rate for fathers $\geq 50$ than for those $\leq 49$ might be 0 thought to result from chance with a probability of $\vec{\infty}$ 0.05 , if one ignores the fact that we performed $\rightarrow$ several tests of significance. On the other hand, such $\mathrm{N}_{\mathrm{N}}$ a result could be produced by some unrecognised bias. In other studies the question of bias has focused in the opposite direction: was the lack of an $\vec{T}$ effect observed in earlier works by one of us ${ }^{4} \stackrel{\mathbb{D}}{O}$ produced by underascertainment of Down syndrome cases with some attendant bias? It was, for example, 㜽 suggested $^{6}$ that data on infants with Down syndrome $\vec{A}$ born to older father-younger mother couples $\infty$ would be preferentially lost. This was proposed under the following schema: Down syndrome ascertainment is lower for babies born to parents of lower social classes, and the average paternal agematernal age difference increases with decreasing social status. We evaluated the rates of Down $\mathbb{Q}$ syndrome from the Norwegian data by two measures $\overrightarrow{\overrightarrow{0}}$ of social status, maternal education and occupation, $\overline{3}$ and found no suggestion of differential ascertainment. $\vec{T}$

Since paternal age was missing more frequently from the certificates of babies born to young mothers than from those born to older mothers, we must also question whether this pattern introduces a $\dot{\sigma}$ bias. We found that the rate of Down syndrome was somewhat higher among those births where paternal $\delta$ age was unknown than among those where it was known. We have no data with which to investigate 의 the possibility that the fathers whose ages were $D$ unknown tended to be older. We do not believe they을 were and feel that most infants whose father's age $\bar{N}$ was unknown were born to unmarried couples con- 5 sisting of young women and young men. In any case, $\mathbb{O}$ we did not find that the contrast between the known and unknown paternal age categories was more pronounced in women $\leq 39$ than in women 40 and over.

With the substantial underascertainment of Down $\stackrel{\mathcal{D}}{\rightarrow}$ syndrome and the attendant potential for bias, one $\square$ mav reasonably ask whether or not it is prudent to 0 use such data as the Norwegian to ask questions $\stackrel{\mathbb{R}}{\circ}$ about a paternal age effect. In this connection we $\overrightarrow{\mathbb{D}}$ should note that a fairly high proportion of fetuses $\frac{\sigma}{0}$ 
with Down syndrome are believed to be aborted spontaneously. Only a fraction of affected conceptions are available for study at birth, even when one has complete ascertainment at this stage of life. Thus, inference about a paternal age effect through the use of cases which survive early pregnancy carries with it a hazard of unknown magnitude. On balance, therefore, we feel justified in using the Norwegian data. If paternal age related biases were non-existent (that is, if the probability of the ascertainment of a Down syndrome case were independent of father's age, conditional on mother's age), or of small magnitude, then these data would be useful in providing some indication of how strong a paternal age effect might be. For example, suppose we knew nothing about the association between maternal age and the risk of Down syndrome. Then the use of the Norwegian data would mislead considerably about the absolute level of the maternal age specific risks. They would, however, mislead only a little if one were interested in the relative risks at different maternal ages. If the same principle applies to the paternal age effect, then we can say that we have found a relatively small effect of father's age, in support of the proposition of Stene $e t a l^{2}$ and Matsunaga et al. $^{3}$

The pattern of paternal age specific rates noted in the figure also deserves consideration in this regard. It was seen that the paternal rates closely followed the maternal age specific rates from the early twenties to the mid-thirties. From the mid-thirties to the mid-forties the paternal age specific rates did not rise as rapidly as the maternal age specific rates and after the mid-forties the divergence was even more pronounced. In order to interpret this pattern, one must consider the correlation between maternal and paternal ages. The demographic pattern noted in table 3 (progressively smaller increases in maternal age as paternal age increases) would then lead one to expect the paternal age specific rates to rise less rapidly than do the maternal age specific rates. This is so, since the effect of maternal age is so preeminent that the rate at a particular paternal age primarily reflects the corresponding mix of maternal age specific risks. Indeed, if the father's age had no independent effect one would expect the paternal age specific rates to stop rising at the point where increases in the father's age are no longer accompanied by increases in the mother's age. The Norwegian data presented in table 3 suggest that this point is located somewhere over the paternal age of 50. The paternal age specific rates shown in the figure are not particularly stable after age 50, even though they were smoothed with a 5-year moving average. They do, however, show some evidence of a very modest continuing rise. Thus this pattern also lends some credence to the notion of a small independent effect of paternal age.

If the increase is modest in relative terms, it is even more modest in absolute terms. Fathers over 50 only contributed about $6 \%$ of all cases of Down syndrome reported in Norway. If, say, $50 \%$ of these were caused by paternal non-disjunction, then a paternal age effect resulting from fathers over $\mathbf{5 0}$ would only contribute $3 \%$ of the total cases. So while it may be important from an aetiological point of view, a paternal age effect would have relatively little impact from the viewpoint of public health.

It was pointed out earlier ${ }^{4}$ that if the paternal age effect is quite small and that if the rate of paternal non-disjunction is quite high (current estimates suggest 20 to $30 \%$ of cases result from this cause), then one might entertain the possibility that the maternal age effect has causes in addition to, or other than, an increasing frequency of non-disjunction with increasing age. An example of such a cause would be an age related decrease in the effectiveness of the screening mechanism of spontaneous abortion. ${ }^{4}$ It is possible that the younger mother is more 'efficient', aborting a higher proportion of affected fetuses than older mothers? This possibility was recently discussed by Sved and Sandler. ${ }^{13}$

\section{References}

1 Magenis RE, Chamberlin RE. Parental origin of nondisjunction. In: de la Cruz FF, Gerald PS, eds. Trisomy 21 (Down syndrome): research perspectives. Baltimore: University Park Press, 1980 (in press).

2 Stene J, Fischer G, Stene E, Mikkelsen M, Petersen E. Paternal age effect in Down's syndrome. Ann Hum Genet 1977;40:299-306.

3 Matsunaga E, Akisa T, Hidetsune O, Kikuchi Y. Reexamination of paternal age effect in Down's syndrome. Hum Genet 1978;40:259-68.

4 Erickson JD. Down syndrome, paternal age, maternal age and birth order. Ann Hum Genet 1978;41:289-98.

5 Erickson JD. Paternal age and Down syndrome. Am J Hum Genet 1979;31:489-97.

- Stene J, Stene E. On data and methods in investigations on parental age effects. Comments on a paper by JD Erickson. Ann Hum Genet 1978;41:465-8.

7 Bjerkedal T, Bakketeig LS. Surveillance of congenital malformations and other conditions of the newborn. Int J Epidemiol 1975;4:31-6.

8 Stene J, Stene E. Statistical methods for detecting a moderate paternal age effect on incidence when a strong maternal one is present. Ann Hum Genet 1977; 40: 343-53.

9 Mantel N, Haenszel W. Statistical aspects of the analysis of data from retrospective studies of disease. $J$ Natl Cancer Inst 1959;22:719-48.

10 Ipsen J, Feigl P. Bancroft's introduction to biostatistics. New York: Harper and Row, 1970.

11 Mantel N. Chi-square tests with one degree of freedom; 
extensions of the Mantel-Haenszel procedure. J Am Stat Assoc 1963;58:690-700.

12 Hook EB, Lindsjö A. Down syndrome in livebirths by single year maternal age interval in a Swedish study: comparison with results from a New York State study. Am J Hum Genet 1978;30:19-27.

13 Sved JA, Sandler L. Does the maternal age effect in Down syndrome necessarily reflect increased nondisjunction.
In: de la Cruz FF, Gerald PS, eds. Trisomy 21 (Down $\stackrel{\mathbb{D}}{-}$ syndrome): research perspectives. Baltimore: University Park Press, 1980 (in press).

Requests for reprints to Dr J D Erickson, Bureau of Epidemiology, Center for Disease Control, Atlanta, Georgia 30333, USA. 